\title{
Some Complete and Intermediate Polynomials in Algebraic Complexity Theory
}

\author{
Meena Mahajan and Nitin Saurabh \\ The Institute of Mathematical Sciences, Chennai, India \\ meena@imsc.res.in, nitin@imsc.res.in
}

\begin{abstract}
We provide a list of new natural VNP-intermediate polynomial families, based on basic (combinatorial) NP-complete problems that are complete under parsimonious reductions. Over finite fields, these families are in VNP, and under the plausible hypothesis $\operatorname{Mod}_{p} \mathrm{P} \nsubseteq \mathrm{P} /$ poly, are neither VNP-hard (even under oracle-circuit reductions) nor in VP. Prior to this, only the Cut Enumerator polynomial was known to be VNP-intermediate, as shown by Bürgisser in 2000.

We next show that over rationals and reals, two of our intermediate polynomials, based on satisfiability and Hamiltonian cycle, are not monotone affine polynomial-size projections of the permanent. This augments recent results along this line due to Grochow.

Finally, we describe a (somewhat natural) polynomial defined independent of a computation model, and show that it is VP-complete under polynomial-size projections. This complements a recent result of Durand et al. (2014) which established VP-completeness of a related polynomial but under constant-depth oracle circuit reductions. Both polynomials are based on graph homomorphisms. A simple restriction yields a family similarly complete for VBP.
\end{abstract}

\section{Introduction}

The algebraic analogue of the $\mathrm{P}$ versus NP problem, famously referred to as the VP versus VNP question, is one of the most significant problem in algebraic complexity theory. Valiant [28] showed that the PERMANENT polynomial is VNP-complete (over fields of char $\neq 2$ ). A striking aspect of this polynomial is that the underlying decision problem, in fact even the search problem, is in P. Given a graph, we can decide in polynomial time whether it has a perfect matching, and if so find a maximum matching in polynomial time [12]. Since the underlying problem is an easier problem, it helped in establishing VNP-completeness of a host of other polynomials by a reduction from the PERMANEnT polynomial (cf. [4]). Inspired from classical results in structural complexity theory, in particular [20], Bürgisser [5] proved that if Valiant's hypothesis (i.e. VP $\neq \mathrm{VNP}$ ) is true, then, over any field there is a $p$ family in VNP which is neither in VP nor VNP-complete with respect to $c$-reductions. Let us call such polynomial families VNP-intermediate (i.e. in VNP, not VNP-complete, not in VP). Further, Bürgisser [5] showed that over finite fields, a specific family of polynomials is VNPintermediate, provided the polynomial hierarchy $\mathrm{PH}$ does not collapse to the second level. On an intuitive level these polynomials enumerate cuts in a graph. This is a remarkable result, when compared with the classical P-NP setting or the BSS-model. Though the existence of problems with intermediate complexity has been established in the latter settings, due to the involved "diagonalization" arguments used to construct them, these problems seem highly unnatural. That is, their definitions are not motivated by an underlying combinatorial problem but guided by the needs of the proof and, hence, seem artificial. The question of 
whether there are other naturally-defined VNP-intermediate polynomials was left open by Bürgisser [4]. We remark that to date the cut enumerator polynomial from [5] is the only known example of a natural polynomial family that is VNP-intermediate.

The question of whether the classes VP and VNP are distinct is often phrased as whether Perm $_{n}$ is not a quasi-polynomial-size projection of Det $_{n}$. The importance of this reformulation stems from the fact that it is a purely algebraic statement, devoid of any dependence on circuits. While we have made very little progress on this question of determinantal complexity of the permanent, the progress in restricted settings has been considerable. One of the success stories in theoretical computer science is unconditional lower bound against monotone computations [24, 25, 1]. In particular, Razborov [25] proved that computing the permanent over the Boolean semiring requires monotone circuits of size at least $n^{\Omega(\log n)}$. Jukna [18] observed that if the Hamilton cycle polynomial is a monotone $p$-projection of the permanent, then, since the clique polynomial is a monotone projection of the Hamiltonian cycle [28, and the clique requires monotone circuits of exponential size [1], one would get a lower bound of $2^{n^{\Omega(1)}}$ for monotone circuits computing the permanent, thus improving on [25]. The importance of this observation is also highlighted by the fact that such a monotone $p$-projection, over the reals, would give an alternate proof of the result of Jerrum and Snir [17] that computing the permanent by monotone circuits over $\mathbb{R}$ requires size at least $2^{n^{\Omega(1)}}$. (Jerrum and Snir [17] proved that the permanent requires monotone circuits of size $2^{\Omega(n)}$ over $\mathbb{R}$ and the tropical semiring.) The first progress on this question raised in [18] was made recently by Grochow [15]. He showed that the Hamiltonian cycle polynomial is not a monotone sub-exponential-size projection of the permanent. This already answered Jukna's question in its entirety, but Grochow [15] used his techniques to further establish that polynomials like the perfect matching polynomial, and even the VNP-intermediate cut enumerator polynomial of Bürgisser [5], are not monotone polynomial-size projections of the permanent. This raises an intriguing question of whether there are other such non-negative polynomials which share this property.

While the Perm vs Det problem has become synonymous with the VP vs VNP question, there is a somewhat unsatisfactory feeling about it. This rises from two facts: one, that the VP-hardness of the determinant is known only under the more powerful quasi-polynomialsize projections, and, second, the lack of natural VP-complete polynomials (with respect to polynomial-size projections) in the literature. (In fact, with respect to $p$-projections, the determinant is complete for the possibly smaller class VBP of polynomial-sized algebraic branching programs.) To remedy this situation, it seems crucial to understand the computation in VP. Bürgisser [4] showed that a generic polynomial family constructed using a topological sort of a generic VP circuit, while controlling the degree, is complete for VP. Raz [23], using the depth reduction of [29], showed that a family of "universal circuits" is VP-complete. Thus both families directly depend on the circuit definition or characterization of VP. Last year, Durand et al. [11] made significant progress and provided a natural, first of its kind, VP-complete polynomial. However, the natural polynomials studied by Durand et al. lacked a bit of punch because their completeness was established under polynomial-size constant depth c-reductions rather than projections. 
In this paper, we make progress on all three fronts. First, we provide a list of new natural polynomial families, based on basic (combinatorial) NP-complete problems [14] whose completeness is via parsimonious reductions [27], that are VNP-intermediate over finite fields (Theorem 1). Then, we show that over reals, some of our intermediate polynomials are not monotone affine polynomial-size projections of the permanent (Theorem 2). As in [15], the lower bound results about monotone affine projections are unconditional. Finally, we improve upon [11] by characterizing VP and establishing a natural VP-complete polynomial under polynomial-size projections (Theorem [6). A modification yields a family similarly complete for VBP (Theorems 7, 8).

Organization of the paper. We give basic definitions in Section 2. Section 3 contains our discussion on intermediate polynomials. In Section 4 we establish lower bounds under monotone affine projections. The discussion on completeness results appears in Section 5. We end in Section 6 with some interesting questions for further exploration.

\section{Preliminaries}

Algebraic complexity: We say that a polynomial $f$ is a projection of $g$ if $f$ can be obtained from $g$ by setting the variables of $g$ to either constants in the field, or to the variables of $f$. A sequence $\left(f_{n}\right)$ is a projection of $\left(g_{m}\right)$, if each $f_{n}$ is a projection of $g_{t}$ for some $t=t(n)$ polynomially bounded in $n$. There are other notions of reductions between families of polynomials, like c-reductions (polynomial-size oracle circuit reductions), constant-depth c-reductions, and linear p-projections. For more on these reductions, see [4].

An arithmetic circuit is a directed acyclic graph with leaves labeled by variables or constants from an underlying field, internal nodes labeled by field operations + and $\times$, and a designated output gate. Each node computes a polynomial in a natural way. The polynomial computed by a circuit is the polynomial computed at its output gate. A parse tree of a circuit captures monomial generation within the circuit. Duplicating gates as needed, unwind the circuit into a formula (fan-out one); a parse tree is a minimal sub-tree (of this unwound formula) that contains the output gate, that contains all children of each included $\times$ gate, and that contains exactly one child of each included + gate. For a complete definition see [21]. A circuit is said to be skew if at every $\times$ gate, at most one incoming edge is the output of another gate.

A family of polynomials $\left(f_{n}\left(x_{1}, \ldots, x_{m(n)}\right)\right)$ is called a $p$-family if both the degree $d(n)$ of $f_{n}$ and the number of variables $m(n)$ are bounded by a polynomial in $n$. A $p$-family is in VP (resp. VBP) if a circuit family (skew circuit family, resp.) $\left(C_{n}\right)$ of size polynomially bounded in $n$ computes it. A sequence of polynomials $\left(f_{n}\right)$ is in VNP if there exist a sequence $\left(g_{n}\right)$ in VP, and polynomials $m$ and $t$ such that for all $n, f_{n}(\bar{x})=\sum_{\bar{y} \in\{0,1\}^{t(\bar{x})}} g_{n}\left(x_{1}, \ldots, x_{m(n)}, y_{1}, \ldots, y_{t(n)}\right)$. (VBP denotes the algebraic analogue of branching programs. Since these are equivalent to skew circuits, we directly use a skew circuit definition of VBP.)

Boolean complexity: We need some basics from Boolean complexity theory. Let P/poly denote the class of languages decidable by polynomial-sized Boolean circuit families. A function 
$\phi:\{0,1\}^{*} \rightarrow \mathbb{N}$ is in \#P if there exists a polynomial $p$ and a polynomial time deterministic Turing machine $M$ such that for all $x \in\{0,1\}^{*}, f(x)=\left|\left\{y \in\{0,1\}^{p(|x|)} \mid M(x, y)=1\right\}\right|$. For a prime $p$, define

$$
\begin{aligned}
\#{ }_{p} \mathrm{P} & =\left\{\psi:\{0,1\}^{*} \rightarrow \mathbb{F}_{p} \mid \psi(x)=\phi(x) \bmod p \text { for some } \phi \in \# \mathrm{P}\right\}, \\
\operatorname{Mod}_{p} \mathrm{P} & =\left\{L \subseteq\{0,1\}^{*} \mid \text { for some } \phi \in \# \mathrm{P}, x \in L \Longleftrightarrow \phi(x) \equiv 1 \bmod p\right\}
\end{aligned}
$$

It is easy to see that if $\phi:\{0,1\}^{*} \rightarrow \mathbb{N}$ is \#P-complete with respect to parsimonious reductions (that is, for every $\psi \in \# P$, there is a polynomial-time computable function $f:\{0,1\}^{*} \rightarrow\{0,1\}^{*}$ such that for all $\left.x \in\{0,1\}^{*}, \psi(x)=\phi(f(x))\right)$, then the language $L=\{x \mid \phi(x) \equiv 1 \bmod p\}$ is $\operatorname{Mod}_{p} \mathrm{P}$-complete with respect to many-one reductions.

Graph Theory: We consider the treewidth and pathwidth parameters for an undirected graph. We will work with a "canonical" form of decompositions which is generally useful in dynamic-programming algorithms.

A (nice) tree decomposition of a graph $G$ is a pair $\mathcal{T}=\left(T,\left\{X_{t}\right\}_{t \in V(T)}\right)$, where $T$ is a tree, rooted at $X_{r}$, whose every node $t$ is assigned a vertex subset $X_{t} \subseteq V(G)$, called a bag, such that the following conditions hold:

1. $X_{r}=\emptyset,\left|X_{\ell}\right|=1$ for every leaf $\ell$ of $T$, and $\cup_{t \in V(T)} X_{t}=V(G)$.

That is, the root contain the empty bag, the leaves contain singleton sets, and every vertex of $G$ is in at least one bag.

2. For every $(u, v) \in E(G)$, there exists a node $t$ of $T$ such that $\{u, v\} \subseteq X_{t}$.

3. For every $u \in V(G)$, the set $T_{u}=\left\{t \in V(T) \mid u \in X_{t}\right\}$ induces a connected subtree of $T$.

4. Every non-leaf node $t$ of $T$ is of one of the following three types:

- Introduce node: $t$ has exactly once child $t^{\prime}$, and $X_{t}=X_{t^{\prime}} \cup\{v\}$ for some vertex $v \notin X_{t^{\prime}}$. We say that $v$ is introduced at $t$.

- Forget node: $t$ has exactly one child $t^{\prime}$, and $X_{t}=X_{t^{\prime}} \backslash\{w\}$ for some vertex $w \in X_{t^{\prime}}$. We say that $w$ is forgotten at $t$.

- Join node: $t$ has two children $t_{1}, t_{2}$, and $X_{t}=X_{t_{1}}=X_{t_{2}}$.

The width of a tree decomposition $\mathcal{T}$ is one less than the size of the largest bag; that is, $\max _{t \in V(T)}\left|X_{t}\right|-1$. The tree-width of a graph $G$ is the minimum possible width of a tree decomposition of $G$.

In a similar way we can also define a nice path decomposition of a graph. For a complete definition we refer to [8].

A sequence $\left(G_{n}\right)$ of graphs is called a $p$-family if the number of vertices in $G_{n}$ is polynomially bounded in $n$. It is further said to have bounded tree(path)-width if for some absolute constant $c$ independent of $n$, the tree(path)-width of each graph in the sequence is bounded by $c$.

A homomorphism from $G$ to $H$ is a map from $V(G)$ to $V(H)$ preserving edges. A graph is called rigid if it has no homomorphism to itself other than the identity map. Two graphs $G$ and $H$ are called incomparable if there are no homomorphisms from $G \rightarrow H$ as well as $H \rightarrow G$. It is known that asymptotically almost all graphs are rigid, and almost all pairs of nonisomorphic graphs are also incomparable. For the purposes of this paper, we only need a collection of three rigid and mutually incomparable graphs. For more details, we refer to [16]. 


\section{VNP-intermediate}

In [5], Bürgisser showed that unless PH collapses to the second level, an explicit family of polynomials, called the cut enumerator polynomial, is VNP-intermediate. He raised the question, recently highlighted again in [15], of whether there are other such natural VNPintermediate polynomials. In this section we show that in fact his proof strategy itself can be adapted to other polynomial families as well. The strategy can be described abstractly as follows: Find an explicit polynomial family $h=\left(h_{n}\right)$ satisfying the following properties.

M: Membership. The family is in VNP.

E: Ease. Over a field $\mathbb{F}_{q}$ of size $q$ and characteristic $p, h$ can be evaluated in $\mathrm{P}$. Thus if $h$ is VNP-hard, then we can efficiently compute \#P-hard functions, modulo $p$.

H: Hardness. The monomials of $h$ encode solutions to a problem that is \#P-hard via parsimonious reductions. Thus if $h$ is in VP, then the number of solutions, modulo $p$, can be extracted using coefficient computation.

Then, unless $\operatorname{Mod}_{p} \mathrm{P} \subseteq \mathrm{P} /$ poly (which in turn implies that $\mathrm{PH}$ collapses to the second level, [19]), $h$ is VNP-intermediate.

We provide a list of $p$-families that, under the same condition $\operatorname{Mod}_{p} \mathrm{P} \nsubseteq \mathrm{P} /$ poly, are VNPintermediate. All these polynomials are based on basic combinatorial NP-complete problems that are complete under parsimonious reduction.

(1) The satisfiablity polynomial Sat ${ }^{q}=\left(\operatorname{Sat}_{n}{ }_{n}\right)$ : For each $n$, let $\mathrm{Cl}_{n}$ denote the set of all possible clauses of size 3 over $2 n$ literals. There are $n$ variables $\tilde{X}=\left\{X_{i}\right\}_{i=1}^{n}$, and also $8 n^{3}$ clause-variables $\tilde{Y}=\left\{Y_{c}\right\}_{c \in \mathrm{Cl}_{n}}$, one for each 3-clause $c$.

$$
\text { Sat }_{n}{ }_{n}:=\sum_{a \in\{0,1\}^{n}}\left(\prod_{i \in[n]: a_{i}=1} X_{i}^{q-1}\right)\left(\prod_{\substack{c \in \mathrm{Cl}_{n} \\ a \text { satisfies } c}} Y_{c}^{q-1}\right) .
$$

For the next three polynomials, we consider the complete graph $G_{n}$ on $n$ nodes, and we have the set of variables $\tilde{X}=\left\{X_{e}\right\}_{e \in E_{n}}$ and $\tilde{Y}=\left\{Y_{v}\right\}_{v \in V_{n}}$.

(2) The vertex cover polynomial $\mathrm{VC}^{\mathrm{q}}=\left(\mathrm{VC}_{n}^{\mathrm{q}}\right)$ :

$$
\mathrm{VC}_{n}^{\mathrm{q}}:=\sum_{S \subseteq V_{n}}\left(\prod_{e \in E_{n}: e \text { is incident on } S} X_{e}^{q-1}\right)\left(\prod_{v \in S} Y_{v}^{q-1}\right) .
$$

(3) The clique/independent set polynomial $\mathrm{CIS}^{\mathrm{q}}=\left(\mathrm{CIS}_{n}{ }_{n}\right)$ :

$$
\mathrm{CIS}_{n}^{\mathrm{q}}:=\sum_{T \subseteq E_{n}}\left(\prod_{e \in T} X_{e}^{q-1}\right)\left(\prod_{v \text { incident on } T} Y_{v}^{q-1}\right) .
$$

(4) The clow polynomial $\operatorname{Clow}^{q}=\left(\operatorname{Clow}_{n}{ }_{n}\right)$ : A clow in an $n$-vertex graph is a closed walk of length exactly $n$, in which the minimum numbered vertex (called the head) appears exactly 
once.

$$
\operatorname{Clow}_{n}{ }_{n}:=\sum_{w: \text { clow of length } n}\left(\prod_{e: \text { edges in } w} X_{e}^{q-1}\right)\left(\prod_{\substack{v: \text { vertices in } w \\(\text { counted only once) }}} Y_{v}^{q-1}\right) .
$$

If an edge $e$ is used $k$ times in a clow, it contributes $X_{e}^{k(q-1)}$ to the monomial. But a vertex $v$ contributes only $Y_{v}^{q-1}$ even if it appears more than once. More precisely,

$$
\left.\operatorname{Clow}_{n}^{\mathrm{q}}:=\sum_{\substack{w=\left\langle v_{0}, v_{1}, \ldots, v_{n-1}\right\rangle: \\ \forall j>0, v_{0}<v_{j}}}\left(\prod_{i \in[n]} X_{\left(v_{i-1}, v_{i} \bmod n\right.}^{q-1}\right)\right)\left(\prod_{v \in\left\{v_{0}, v_{1}, \ldots, v_{n-1}\right\}} Y_{v}^{q-1}\right) .
$$

(5) The 3D-matching polynomial 3DM ${ }^{\mathrm{q}}=\left(3 \mathrm{DM}_{n}{ }_{n}\right)$ : Consider the complete tripartite hypergraph, where each part in the partition $\left(A_{n}, B_{n}, C_{n}\right)$ contain $n$ nodes, and each hyperedge has exactly one node from each part. We have variables $X_{e}$ for hyperedge $e$ and $Y_{v}$ for node $v$.

$$
3 \mathrm{DM}_{n}^{\mathrm{q}}:=\sum_{M \subseteq A_{n} \times B_{n} \times C_{n}}\left(\prod_{e \in M} X_{e}^{q-1}\right)\left(\prod_{\substack{v \in M \\ \text { (counted only once) }}} Y_{v}^{q-1}\right) .
$$

We show that if $\operatorname{Mod}_{p} \mathrm{P} \nsubseteq \mathrm{P} /$ poly, then all five polynomials defined above are VNPintermediate.

Theorem 1. Over a finite field $\mathbb{F}_{q}$ of characteristic $p$, the polynomial families Sat ${ }^{\mathrm{q}}$, VCq, $\mathrm{CIS}^{\mathrm{q}}, \mathrm{Clow}^{\mathrm{q}}$, and $3 \mathrm{DM}^{\mathrm{q}}$, are in VNP. Further, if $\operatorname{Mod}_{p} \mathrm{P} \nsubseteq \mathrm{P} /$ poly, then they are all VNPintermediate; that is, neither in VP nor VNP-hard with respect to c-reductions.

Proof. (M) An easy way to see membership in VNP is to use Valiant's criterion ([28]; see also Proposition 2.20 in [4]); the coefficient of any monomial can be computed efficiently, hence the polynomial is in VNP. This establishes membership for all families.

We first illustrate the rest of the proof by showing that the polynomial Sat ${ }^{q}$ satisfies the properties $(\mathrm{H}),(\mathrm{E})$.

$(\mathrm{H})$ : Assume $\left(\right.$ Sat $_{n}{ }_{n}$ ) is in VP, via polynomial-sized circuit family $\left\{C_{n}\right\}_{n \geq 1}$. We will use $C_{n}$ to give a $\mathrm{P} /$ poly upper bound for computing the number of satisfying assignments of a 3-CNF formula, modulo $p$. Since this question is complete for $\operatorname{Mod}_{p} \mathrm{P}$, the upper bound implies $\operatorname{Mod}_{p} P$ is in $\mathrm{P} /$ poly.

Given an instance $\phi$ of 3SAT, with $n$ variables and $m$ clauses, consider the projection of Sat ${ }_{n}$ obtained by setting all $Y_{c}$ for $c \in \phi$ to $t$, and all other variables to 1 . This gives the polynomial Sat ${ }^{\mathrm{q}} \phi(t)=\sum_{j=1}^{m} d_{j} t^{j(q-1)}$ where $d_{j}$ is the number of assignments (modulo $p$ ) that satisfy exactly $j$ clauses in $\phi$. Our goal is to compute $d_{m}$.

We convert the circuit $C$ into a circuit $D$ that compute elements of $\mathbb{F}_{q}[t]$ by explicitly giving their coefficient vectors, so that we can pull out the desired coefficient. (Note that after the projection described above, $C$ works over the polynomial ring $\mathbb{F}_{q}[t]$.) Since the polynomial 
computed by $C$ is of degree $m(q-1)$, we need to compute the coefficients of all intermediate polynomials too only upto degree $m(q-1)$. Replacing + by gates performing coordinate-wise addition, $\times$ by a sub-circuit performing (truncated) convolution, and supplying appropriate coefficient vectors at the leaves gives the desired circuit. Since the number of clauses, $m$, is polynomial in $n$, the circuit $D$ is also of polynomial size. Given the description of $C$ as advice, the circuit $D$ can be evaluated in $\mathrm{P}$, giving a $\mathrm{P} /$ poly algorithm for computing \#3-SAT $(\phi)$ $\bmod p$. Hence $\operatorname{Mod}_{p} \mathrm{P} \subseteq \mathrm{P} /$ poly.

(E) Consider an assignment to $\tilde{X}$ and $\tilde{Y}$ variables in $\mathbb{F}_{q}$. Since all exponents are multiples of $(q-1)$, it suffices to consider $0 / 1$ assignments to $\tilde{X}$ and $\tilde{Y}$. Each assignment a contributes 0 or 1 to the final value; call it a contributing assignment if it contributes 1 . So we just need to count the number of contributing assignments. An assignment $a$ is contributing exactly when $\forall i \in[n], X_{i}=0 \Longrightarrow a_{i}=0$, and $\forall c \in \mathrm{Cl}_{n}, Y_{c}=0 \Longrightarrow a$ does not satisfy $c$. These two conditions, together with the values of the $X$ and $Y$ variables, constrain many bits of a contributing assignment; an inspection reveals how many (and which) bits are so constrained. If any bit is constrained in conflicting ways (for example, $X_{i}=0$, and $Y_{c}=0$ for some clause $c$ containing the literal $\bar{x}_{i}$ ), then no assignment is contributing (either $a_{i}=1$ and the $X$ part becomes zero due to $X_{i}^{a_{i}}$, or $a_{i}=0$ and the $Y$ part becomes zero due to $Y_{c}$ ). Otherwise, some bits of a potentially contributing assignment are constrained by $X$ and $Y$, and the remaining

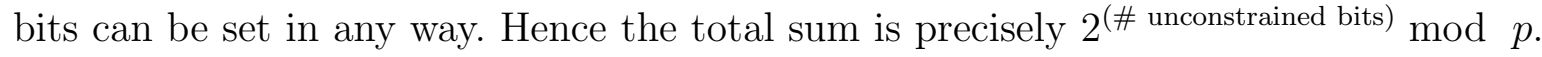

Now assume Sat ${ }^{q}$ is VNP-hard. Let $L$ be any language in $\operatorname{Mod}_{p} P$, witnessed via \#Pfunction $f$. (That is, $x \in L \Longleftrightarrow f(x) \equiv 1 \bmod p$.) By the results of [ㅁ, 4], there exists a $p$-family $r=\left(r_{n}\right) \in \mathrm{VNP}_{\mathbb{F}_{p}}$ such that $\forall n, \forall x \in\{0,1\}^{n}, r_{n}(x)=f(x) \bmod p$. By assumption, there is a $c$-reduction from $r$ to Sat ${ }^{q}$. We use the oracle circuits from this reduction to decide instances of $L$. On input $x$, the advice is the circuit $C$ of appropriate size reducing $r$ to Sat ${ }^{q}$. We evaluate this circuit bottom-up. At the leaves, the values are known. At + and $\times$ gates, we perform these operations in $\mathbb{F}_{q}$. At an oracle gate, the paragraph above tells us how to evaluate the gate. So the circuit can be evaluated in polynomial time, showing that $L$ is in $\mathrm{P} /$ poly. Thus $\operatorname{Mod}_{p} \mathrm{P} \subseteq \mathrm{P} /$ poly.

For the other four families, it suffices to show the following, since the rest is identical as for Sat ${ }^{q}$.

H'. The monomials of $h$ encode solutions to a problem that is \#P-hard via parsimonious reductions.

E'. Over $\mathbb{F}_{q}, h$ can be evaluated in $\mathrm{P}$.

We describe this for the polynomial families one by one.

\section{The vertex cover polynomial $\mathrm{VC}^{\mathrm{q}}=\left(\mathrm{VC}_{n}\right)$ :}

$$
\mathrm{VC}_{n}^{\mathrm{q}}:=\sum_{S \subseteq V_{n}}\left(\prod_{e \in E_{n}: e \text { is incident on } S} X_{e}^{q-1}\right)\left(\prod_{v \in S} Y_{v}^{q-1}\right) .
$$

(H'): Given an instance of vertex cover $A=(V(A), E(A))$ such that $|V(A)|=n$ and $|E(A)|=$ $m$, we show how $\mathrm{VC}^{\mathrm{q}}{ }_{n}$ encodes the number of solutions of instance $A$. Consider the following 
projection of $\mathrm{VC}_{n}$. Set $Y_{v}=t$, for $v \in V(A)$. For $e \in E(A)$, set $X_{e}=z$; otherwise $e \notin E(A)$ and set $X_{e}=1$. Thus, we have

$$
\mathrm{VC}_{n}^{\mathrm{q}}(z, t)=\sum_{S \subseteq V_{n}} z^{(\# \text { edges incident on } S)(q-1)} t^{|S|(q-1)} .
$$

Hence, it follows that the number of vertex cover of size $k$, modulo $p$, is the coefficient of $z^{m(q-1)} t^{k(q-1)}$ in $\operatorname{VC}_{n}(z, t)$

(E'): Consider the weighted graph given by the values of $\tilde{X}$ and $\tilde{Y}$ variables. Each subset $S \subseteq V_{n}$ contributes 0 or 1 to the total. A subset $S \subseteq V_{n}$ contributes 1 to VCq $_{n}$ if and only if every vertex in $S$ has non-zero weight, and every edge incident on each vertex in $S$ has non-zero weight. That is, $S$ is a subset of full-degree vertices. Therefore, the total sum is $2^{\text {(\# full-degree vertices) }} \bmod p$.

The clique/independent set polynomial $\operatorname{CIS}^{q}=\left(\operatorname{CIS}_{n}\right)$ :

$$
\mathrm{CIS}_{n}^{\mathrm{q}}:=\sum_{T \subseteq E_{n}}\left(\prod_{e \in T} X_{e}^{q-1}\right)\left(\prod_{v \text { incident on } T} Y_{v}^{q-1}\right) .
$$

(H'): Given an instance of clique $A=(V(A), E(A))$ such that $|V(A)|=n$ and $|E(A)|=m$, we show how $\mathrm{CIS}_{n}$ encodes the number of solutions of instance $A$. Consider the following projection of $\mathrm{CIS}_{n}{ }_{n}$. Set $Y_{v}=t$, for $v \in V(A)$. For $e \in E(A)$, set $X_{e}=z$; otherwise $e \notin E(A)$ and set $X_{e}=1$. (This is the same projection as used for vertex cover.) Thus, we have

$$
\mathrm{CIS}_{n}^{\mathrm{q}}(z, t)=\sum_{T \subseteq E_{n}} z^{|T \cap E(A)|(q-1)} t^{(\# \text { vertices incident on } T)(q-1)} .
$$

Now it follows easily that the number of cliques of size $k$, modulo $p$, is the coefficient of $z^{\left(\begin{array}{c}k \\ 2\end{array}\right)(q-1)} t^{k(q-1)}$ in $\operatorname{CIS}_{n}(z, t)$.

(E'): Consider the weighted graph given by the values of $\tilde{X}$ and $\tilde{Y}$ variables. Each subset $T \subseteq E_{n}$ contributes 0 or 1 to the sum. A subset $T \subseteq E_{n}$ contributes 1 to the sum if and only if all edges in $T$ have non-zero weight, and every vertex incident on $T$ must have nonzero weight. Therefore, we consider the graph induced on vertices with non-zero weights. Any subset of edges in this induced graph contributes 1 to the total sum; all other subsets contribute 0 . Let $\ell$ be the number of edges in the induced graph with non-zero weights. Thus, the total sum is $2^{\ell} \bmod p$.

The clow polynomial Clow $^{\mathbf{q}}=\left(\operatorname{Clow}_{n}\right)$ : A clow in an $n$-vertex graph is a closed walk of length exactly $n$, in which the minimum numbered vertex (called the head) appears exactly once.

$$
\operatorname{Clow}_{n}^{\mathrm{q}}:=\sum_{w: \text { clow of length } n}\left(\prod_{e: \text { edges in } w} X_{e}^{q-1}\right)\left(\prod_{\substack{v: \text { vertices in } w \\ \text { (counted only once })}} Y_{v}^{q-1}\right) .
$$


(If an edge $e$ is used $k$ times in a clow, it contributes $X_{e}^{k(q-1)}$ to the monomial.)

$\left(\mathrm{H}^{\prime}\right)$ : Given an instance $A=(V(A), E(A))$ of the Hamiltonian cycle problem with $|V(A)|=n$ and $|E(A)|=m$, we show how Clow $_{n}{ }_{n}$ encodes the number of Hamiltonian cycles in $A$. Consider the following projection of $\mathrm{Clow}_{n}{ }_{n}$. Set $Y_{v}=t$, for $v \in V(A)$. For $e \in E(A)$, set $X_{e}=z$; otherwise $e \notin E(A)$ and set $X_{e}=1$. (The same projection was used for VCa and CISq.) Thus, we have

$$
\operatorname{Clow}_{n}^{\mathrm{q}}(z, t)=\sum_{w: \text { clow of length } n}\left(\prod_{e: \text { edges in } w \cap E(A)} z^{q-1}\right)\left(\prod_{\substack{\text { vertices in } w \\ \text { (counted only once })}} t^{q-1}\right) .
$$

From the definition, it now follows that number of Hamiltonian cycles in $A$, modulo $p$, is the coefficient of $z^{n(q-1)} t^{n(q-1)}$.

(E'): To evaluate $\operatorname{Clow}_{n}{ }_{n}$ on instantiations of $\tilde{X}$ and $\tilde{Y}$ variables, we consider the weighted graph given by the values to the variables. We modify the edge weights as follows: if an edge is incident on a node with zero weight, we make its weight 0 irrespective of the value of the corresponding $X$ variable. Thus, all zero weight vertices are isolated in the modified graph $G$. Hence, the total sum is equal to the number of closed walks of length $n$, modulo $p$, in this modified graph. This can be computed in polynomial time using matrix powering as follows: Let $G_{i}$ denote the induced subgraph of $G$ with vertices $\{i, \ldots, n\}$, and let $A_{i}$ be its adjacency matrix. We represent $A_{i}$ as an $n \times n$ matrix with the first $i-1$ rows and columns having only zeroes. Now the number of clows with head $i$ is given by the $[i, i]$ entry of $A_{i} A_{i+1}^{n-2} A_{i}$.

The $3 D$-matching polynomial $3 \mathrm{DM}^{\mathbf{q}}=\left(3 \mathrm{DM}_{n}^{\mathrm{q}}\right)$ : Consider the complete tripartite hyper-graph, where each partition contain $n$ nodes, and each hyperedge has exactly one node from each part. As before, there are variables $X_{e}$ for hyperedge $e$ and $Y_{v}$ for node $v$.

$$
3 \mathrm{DM}_{n}^{\mathrm{q}}:=\sum_{M \subseteq A_{n} \times B_{n} \times C_{n}}\left(\prod_{e \in M} X_{e}^{q-1}\right)\left(\prod_{\substack{v \in M \\ \text { (counted only once) }}} Y_{v}^{q-1}\right) .
$$

$\left(\mathrm{H}^{\prime}\right)$ : Given an instance of 3D-Matching $\mathcal{H}$, we consider the usual projection. The variables corresponding to the vertices are all set to $t$. The edges present in $\mathcal{H}$ are all set to $z$, and the ones not present are set to 1 . Then the number of 3D-matchings in $\mathcal{H}$, modulo $p$, is equal to the coefficient of $z^{n(q-1)} t^{3 n(q-1)}$ in $3 \mathrm{DM}^{\mathrm{q}}{ }_{n}(z, t)$.

$\left(\mathrm{E}^{\prime}\right)$ : To evaluate $3 \mathrm{DM}^{\mathrm{q}}{ }_{n}$ over $\mathbb{F}_{q}$, consider the hypergraph obtained after removing the vertices with zero weight, edges with zero weight, and edges that contain a vertex with zero weight (even if the edges themselves have non-zero weight). Every subset of hyperedges in this modified hypergraph contributes 1 to the total sum, and all other subsets contribute 0 .

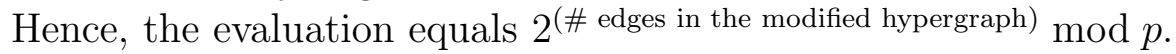

It is worth noting that the cut enumerator polynomial Cut ${ }^{q}$, showed by Bürgisser to be VNP-intermediate over field $\mathbb{F}_{q}$, is in fact VNP-complete over the rationals when $q=2$, 9]. Thus the above technique is specific to finite fields. 


\section{Monotone projection lower bounds}

We now show that some of our intermediate polynomials are not monotone p-projections of the Permanent polynomial. The results here are motivated by the recent results of Grochow [15]. Recall that a polynomial $f\left(x_{1}, \ldots, x_{n}\right)$ is a projection of a polynomial $g\left(y_{1}, \ldots, y_{m}\right)$ if $f\left(x_{1}, \ldots, x_{n}\right)=g\left(a_{1}, \ldots, a_{m}\right)$, where $a_{i}$ 's are either constants or $x_{j}$ for some $j$. The polynomial $f$ is an affine projection of $g$ if $f$ can be obtained from $g$ by replacing each $y_{i}$ with an affine linear function $\ell_{i}(\tilde{x})$. Over any subring of $\mathbb{R}$, or more generally any totally ordered semi-ring, a monotone projection is a projection in which all constants appearing in the projection are non-negative. We say that the family $\left(f_{n}\right)$ is a (monotone affine) projection of the family $\left(g_{n}\right)$ with blow-up $t(n)$ if for all sufficiently large $n, f_{n}$ is a (monotone affine) projection of $g_{t(n)}$.

Theorem 2. Over the reals (or any totally ordered semi-ring), for any $q$, the families Sat ${ }^{\mathrm{q}}$ and $\mathrm{Clow}^{\mathrm{q}}$ are not monotone affine p-projections of the PERMANENT family. Any monotone affine projection from PERMANENT to Sat ${ }^{q}$ must have a blow-up of at least $2^{\Omega(\sqrt{n})}$. Any monotone affine projection from PERMANENT to Clow ${ }^{q}$ must have a blow-up of at least $2^{\Omega(n)}$.

Before giving the proof, we set up some notation. For more details, see [2, 26, 15]. For any polynomial $p$ in $n$ variables, let $\operatorname{Newt}(p)$ denote the polytope in $\mathbb{R}^{n}$ that is the convex hull of the vectors of exponents of monomials of $p$. For any Boolean formula $\phi$ on $n$ variables, let p-SAT $(\phi)$ denote the polytope in $\mathbb{R}^{n}$ that is the convex hull of all satisfying assignments of $\phi$. Let $K_{n}=\left(V_{n}, E_{n}\right)$ denote the $n$-vertex complete graph. The travelling salesperson (TSP) polytope is defined as the convex hull of the characteristic vectors of all subsets of $E_{n}$ that define a Hamiltonian cycle in $K_{n}$.

For a polytope $P$, let $\mathrm{c}(P)$ denote the minimal number of linear inequalities needed to define $P$. A polytope $Q \subseteq \mathbb{R}^{m}$ is an extension of $P \subseteq \mathbb{R}^{n}$ if there is an affine linear map $\pi: \mathbb{R}^{m} \rightarrow \mathbb{R}^{n}$ such that $\pi(Q)=P$. The extension complexity of $P$, denoted $\times c(P)$, is the minimum size $c(Q)$ of any extension $Q$ (of any dimension) of $P$.

The following are straightforward, see for instance [15, 13].

Fact 3 1. c $\left(\right.$ Newt $\left(\right.$ Perm $\left.\left._{n}\right)\right) \leqslant 2 n$.

2. If polytope $Q$ is an extension of polytope $P$, then $x c(P) \leqslant \mathrm{xc}(Q)$.

We use the following recent results.

Proposition 1. 1. Let $f\left(x_{1}, \ldots, x_{n}\right)$ and $g\left(y_{1}, \ldots, y_{m}\right)$ be polynomials over a totally ordered semi-ring $R$, with non-negative coefficients. If $f$ is a monotone projection of $g$, then the intersection of $\operatorname{Newt}(g)$ with some linear subspace is an extension of $\operatorname{Newt}(f)$. In particular, $\mathrm{xc}(\operatorname{Newt}(f)) \leqslant m+\mathrm{c}(\operatorname{Newt}(g))$. [15]

2. For every $n$ there exists a 3SAT formula $\phi$ with $O(n)$ variables and $O(n)$ clauses such that $\mathrm{xc}(\mathrm{p}-\mathrm{SAT}(\phi)) \geqslant 2^{\Omega(\sqrt{n})}$. [2]

3. The extension complexity of the TSP polytope is $2^{\Omega(n)}$. [26] 
Proof. (of Theorem 2.) Let $\phi$ be a 3SAT formula with $n$ variables and $m$ clauses as given by Proposition 1 (2). For the polytope $P=$ p-SAT $(\phi), \mathrm{xc}(P)$ is high.

Let $Q$ be the Newton polytope of Sat ${ }_{n}$. It resides in $N$ dimensions, where $N=n+$ $\left|\mathrm{Cl}_{n}\right|=n+8 n^{3}$, and is the convex hull of vectors of the form $(q-1)\langle\tilde{a} \tilde{b}\rangle$ where $\tilde{a} \in\{0,1\}^{n}$, $\tilde{b} \in\{0,1\}^{N-n}$, and for all $c \in \mathrm{Cl}_{n}, \tilde{a}$ satisfies $c$ if and only if $b_{c}=1$. For each $\tilde{a} \in\{0,1\}^{n}$, there is a unique $\tilde{b} \in\{0,1\}^{N-n}$ such that $(q-1)\langle\tilde{a} \tilde{b}\rangle$ is in $Q$.

Define the polytope $R$, also in $N$ dimensions, to be the convex hull of vectors that are vertices of $Q$ and also satisfy the constraint $\sum_{c \in \phi} b_{c} \geq m$. This constraint discards vertices of $Q$ where $\tilde{a}$ does not satisfy $\phi$. Thus $R$ is an extension of $P$ (projecting the first $n$ coordinates of points in $R$ gives a $(q-1)$-scaled version of $P)$, so by Fact $3(2), \operatorname{xc}(P) \leq \mathrm{xc}(R)$. Further, we can obtain an extension of $R$ from any extension of $Q$ by adding just one inequality; hence $\mathrm{xc}(R) \leq 1+\mathrm{xc}(Q)$.

Suppose Sat ${ }^{\mathrm{q}}$ is a monotone affine projection of Perm ${ }_{n}$ with blow-up $t(n)$. By Fact 3(1) and Proposition 1(1), xc $\left(\operatorname{Newt}\left(\operatorname{Sat}^{q}\right)\right)=\mathrm{xc}(Q) \leq t(n)+c\left(\operatorname{Perm}_{t(n)}\right) \leq O(t(n))$. From the preceding discussion and by Proposition $1(2)$, we get $2^{\Omega(\sqrt{n})} \leq \mathrm{xc}(P) \leq \mathrm{xc}(R) \leq 1+\mathrm{xc}(Q) \leq O(t(n))$. It follows that $t(n)$ is at least $2^{\Omega(\sqrt{n})}$.

For the Clow ${ }^{q}$ polynomial, let $P$ be the TSP polytope and $Q$ be Newt $\left(\mathrm{Clow}^{q}\right)$. The vertices of $Q$ are of the form $(q-1) \tilde{a} \tilde{b}$ where $\tilde{a} \in\{0,1\}^{\left(\begin{array}{c}n \\ 2\end{array}\right)}$ picks a subset of edges, $\tilde{b} \in\{0,1\}^{n}$ picks a subset of vertices, and the picked edges form a length- $n$ clow touching exactly the picked vertices. Define polytope $R$ by discarding vertices of $Q$ where $\sum_{i \in[n]} b_{i}<n$. Now the same argument as above works, using Proposition 1(3) instead of (4).

\section{Complete families for VP and VBP}

The quest for a natural VP-complete polynomial has generated a significant amount of research [4, 23, 22, 7, 11]. The first success story came from [11], where some naturally defined homomorphism polynomials were studied, and a host of them were shown to be complete for the class VP. But the results came with minor caveats. When the completeness was established under projections, there were non-trivial restrictions on the set of homomorphisms $\mathcal{H}$, and sometimes even on the target graph $H$. On the other hand, when all homomorphisms were allowed, completeness could only be shown under seemingly more powerful reductions, namely, constant-depth $c$-reductions. Furthermore, the graphs were either directed or had weights on nodes. It is worth noting that the reductions in [11] actually do not use the full power of generic constant-depth $c$-reductions; a closer analysis reveals that they are in fact linear p-projection. That is, the reductions are linear combinations of polynomially many $p$-projections (see Chapter 3, [4]). Still, this falls short of $p$-projections.

In this work, we remove all such restrictions and show that there is a simple explicit homomorphism polynomial family that is complete for VP under $p$-projections. In this family, the source graphs $G$ are specific bounded-tree-width graphs, and the target graphs $H$ are complete graphs. We also show that a similar family with bounded-path-width source graphs is complete for VBP under $p$-projections. Thus, homomorphism polynomials are rich enough to characterise computations by circuits as well as algebraic branching programs. 
The polynomials we consider are defined formally as follows.

Definition 4 Let $G=(V(G), E(G))$ and $H=(V(H), E(H))$ be two graphs. Consider the set of variables $\bar{Z}:=\left\{Z_{u, a} \mid u \in V(G)\right.$ and $\left.a \in V(H)\right\}$ and $\bar{Y}:=\left\{Y_{(u, v)} \mid(u, v) \in E(H)\right\}$. Let $\mathcal{H}$ be a set of homomorphisms from $G$ to $H$. The homomorphism polynomial $f_{G, H, \mathcal{H}}$ in the variable set $\bar{Y}$, and the generalised homomorphism polynomial $\hat{f}_{G, H, \mathcal{H}}$ in the variable set $\bar{Z} \cup \bar{Y}$, are defined as follows:

$$
\begin{aligned}
f_{G, H, \mathcal{H}} & =\sum_{\phi \in \mathcal{H}}\left(\prod_{(u, v) \in E(G)} Y_{(\phi(u), \phi(v))}\right) . \\
\hat{f}_{G, H, \mathcal{H}} & =\sum_{\phi \in \mathcal{H}}\left(\prod_{u \in V(G)} Z_{u, \phi(u)}\right)\left(\prod_{(u, v) \in E(G)} Y_{(\phi(u), \phi(v))}\right) .
\end{aligned}
$$

Let Hom denote the set of all homomorphisms from $G$ to $H$. If $\mathcal{H}$ equals $\mathbf{H o m}$, then we drop it from the subscript and write $f_{G, H}$ or $\hat{f}_{G, H}$.

Note that for every $G, H, \mathcal{H}, f_{G, H, \mathcal{H}}(\bar{Y})$ equals $\left.\hat{f}_{G, H, \mathcal{H}}(\bar{Y})\right|_{\bar{Z}=\overline{1}}$. Thus upper bounds for $\hat{f}$ give upper bounds for $f$, while lower bounds for $f$ give lower bounds for $\hat{f}$.

We show in Theorem 5 that for any $p$-family $\left(H_{m}\right)$, and any bounded tree-width (pathwidth, respectively) $p$-family $\left(G_{m}\right)$, the polynomial family $\left(f_{m}\right)$ where $f_{m}=\hat{f}_{G_{m}, H_{m}}$ is in VP (VBP, respectively). We then show in Theorem 6 that for a specific bounded tree-width family $\left(G_{m}\right)$, and for $H_{m}=K_{m^{6}}$, the polynomial family $\left(f_{G_{m}, H_{m}}\right)$ is hard, and hence complete, for VP with respect to projections. An analogous statement is shown in Theorem 7 for a specific bounded path-width family $\left(G_{m}\right)$ and for $H_{m}=K_{m^{2}}$. Over fields of characteristic other than 2, VBP-hardness is obtained for a simpler family of source graphs $G_{m}$, as described in Theorem 8 .

\subsection{Upper Bound}

In [11, it was shown that the homomorphism polynomial $\hat{f}_{T_{m}, K_{n}}$ where $T_{m}$ is a binary tree on $m$ leaves, and $K_{n}$ is a complete graph on $n$ nodes, is computable by an arithmetic circuit of size $O\left(m^{3} n^{3}\right)$. Their proof idea is based on recursion: group the homomorphisms based on where they map the root of $T_{m}$ and its children, and recursively compute the sub-polynomials within each group. The sub-polynomials of a specific group have a special set of variables in their monomials. Hence, the homomorphism polynomial can be computed by suitably combining partial derivatives of the sub-polynomials. The partial derivatives themselves can be computed efficiently using the technique of Baur and Strassen, [3].

Generalizing the above idea to polynomials where the source graph is not a binary tree $T_{m}$ but a bounded tree-width graph $G_{m}$ seems hard. The very first obstacle we encounter is to generalize the concept of partial derivative to monomial extension. Combining subpolynomials to obtain the original polynomial also gets rather complicated. 
We sidestep this difficulty by using a dynamic programming approach [10] based on a "nice" tree decomposition of the source graph. This shows that the homomorphism polynomial $\hat{f}_{G, H}$ is computable by an arithmetic circuit of size at most $2|V(G)| \cdot|V(H)|^{t w(G)+1}$. $(2|V(H)|+2|E(H)|)$, where $t w(G)$ is the tree-width of $G$.

Let $\mathcal{T}=\left(T,\left\{X_{t}\right\}_{t \in V(T)}\right)$ be a nice tree decomposition of $G$ of width $\tau$. For each $t \in V(T)$, let $M_{t}=\left\{\phi \mid \phi: X_{t} \rightarrow V(H)\right\}$ be the set of all mappings from $X_{t}$ to $V(H)$. Since $\left|X_{t}\right| \leqslant \tau+1$, we have $\left|M_{t}\right| \leqslant|V(H)|^{\tau+1}$. For each node $t \in V(T)$, let $T_{t}$ be the subtree of $T$ rooted at node $t, V_{t}:=\bigcup_{t^{\prime} \in V\left(T_{t}\right)} X_{t^{\prime}}$, and $G_{t}:=G\left[V_{t}\right]$ be the subgraph of $G$ induced on $V_{t}$. Note that $G_{r}=G$.

We will build the circuit inductively. For each $t \in V(T)$ and $\phi \in M_{t}$, we have a gate $\langle t, \phi\rangle$ in the circuit. Such a gate will compute the homomorphism polynomial from $G_{t}$ to $H$ such that the mapping of $X_{t}$ in $H$ is given by $\phi$. For each such gate $\langle t, \phi\rangle$ we introduce another gate $\langle t, \phi\rangle^{\prime}$ which computes the "partial derivative" (or, quotient) of the polynomial computed at $\langle t, \phi\rangle$ with respect to the monomial given by $\phi$. As we mentioned before, the construction is inductive, starting at the leaf nodes and proceeding towards the root.

Base case (Leaf nodes): Let $\ell \in V(T)$ be a leaf node. Then, $X_{\ell}=\{u\}$ such that $u \in V(G)$. Note that any $\phi \in M_{\ell}$ is just a mapping of $u$ to some node in $V(H)$. Hence, the set $M_{\ell}$ can be identified with $V(H)$. Therefore, for all $h \in V(H)$, we label the gate $\langle\ell, h\rangle$ by the variable $Z_{u, h}$. The derivative gate $\langle\ell, h\rangle^{\prime}$ in this case is set to 1 .

Introduce nodes: Let $t \in V(T)$ be an introduce node, and $t^{\prime}$ be its unique child. Then, $X_{t} \backslash X_{t^{\prime}}=\{u\}$ for some $u \in V(G)$. Let $N(u):=\left\{v \mid v \in X_{t^{\prime}}\right.$ and $\left.(v, u) \in E\left(G_{t}\right)\right\}$. Note that there is a one-to-one correspondence between $\phi \in M_{t}$ and pairs $\left(\phi^{\prime}, h\right) \in M_{t^{\prime}} \times V(H)$. Therefore, for all $\phi\left(=\left(\phi^{\prime}, h\right)\right) \in M_{t}$ such that $\forall v \in N(u),\left(\phi^{\prime}(v), h\right) \in E(H)$, we set

$$
\begin{aligned}
\langle t, \phi\rangle & :=Z_{u, h} \cdot\left(\prod_{v \in N(u)} Y_{\left(\phi^{\prime}(v), h\right)}\right) \cdot\left\langle t^{\prime}, \phi^{\prime}\right\rangle \quad \text { and }, \\
\langle t, \phi\rangle^{\prime} & :=\left\langle t^{\prime}, \phi^{\prime}\right\rangle^{\prime}
\end{aligned}
$$

otherwise we set $\langle t, \phi\rangle=\langle t, \phi\rangle^{\prime}:=0$.

Forget nodes: Let $t \in V(T)$ be a forget node and $t^{\prime}$ be its unique child. Then, $X_{t^{\prime}} \backslash X_{t}=\{u\}$ for some $u \in V(G)$. Again note that there is a one-to-one correspondence between pairs $(\phi, h) \in M_{t} \times V(H)$ and $\phi^{\prime} \in M_{t^{\prime}}$. Let $N(u):=\left\{v \mid v \in X_{t}\right.$ and $\left.(v, u) \in E\left(G_{t^{\prime}}\right)\right\}$. Therefore, for all $\phi \in M_{t}$, we set

$$
\begin{aligned}
&\langle t, \phi\rangle:=\sum_{h \in V(H)}\left\langle t^{\prime},(\phi, h)\right\rangle \quad \text { and } \\
&\langle t, \phi\rangle^{\prime}:=\sum_{\substack{h \in V(H) \\
\forall v \in N(u),(\phi(v), h) \in E(H)}} Z_{u, h} \cdot\left(\prod_{v \in N(u)} Y_{(\phi(v), h)}\right) \cdot\left\langle t^{\prime},(\phi, h)\right\rangle^{\prime} .
\end{aligned}
$$


Join nodes: Let $t \in V(T)$ be a join node, and $t_{1}$ and $t_{2}$ be its two children; we have $X_{t}=X_{t_{1}}=X_{t_{2}}$. Then, for all $\phi \in M_{t}$, we set

$$
\begin{aligned}
\langle t, \phi\rangle & :=\left\langle t_{1}, \phi\right\rangle \cdot\left\langle t_{2}, \phi\right\rangle^{\prime}\left(=\left\langle t_{1}, \phi\right\rangle^{\prime} \cdot\left\langle t_{2}, \phi\right\rangle\right) \\
\langle t, \phi\rangle^{\prime} & :=\left\langle t_{1}, \phi\right\rangle^{\prime} \cdot\left\langle t_{2}, \phi\right\rangle^{\prime} .
\end{aligned}
$$

The output gate of the circuit is $\langle r, \emptyset\rangle$. The correctness of the algorithm is readily seen via induction in a similar way. The bound on the size also follows easily from the construction.

We observe some properties of our construction. First, the circuit constructed is a constantfree circuit. This was the case with the algorithm from [11] too. Second, if we start with a path decomposition, we obtain skew circuits, since the join nodes are absent. The algorithm from [11] does not give skew circuits when $T_{m}$ is a path. (It seems the obstacle there lies in computing partial-derivatives using skew circuits.)

From the above algorithm and its properties, we obtain the following theorem.

Theorem 5. Consider the family of homomorphism polynomials $\left(f_{m}\right)$, where $f_{m}=f_{G_{m}, H_{m}}(\bar{Z}, \bar{Y})$, and $\left(H_{m}\right)$ is a p-family of complete graphs.

- If $\left(G_{m}\right)$ is a p-family of graphs of bounded tree-width, then $\left(f_{m}\right) \in \mathrm{VP}$.

- If $\left(G_{m}\right)$ is a p-family of graphs of bounded path-width, then $\left(f_{m}\right) \in \mathrm{VBP}$.

\section{$5.2 \quad$ VP-completeness}

We now turn our attention towards establishing VP-hardness of the homomorphism polynomials. We need to show that there exists a $p$-family $\left(G_{m}\right)$ of bounded tree-width graphs such that $\left(f_{G_{m}, H_{m}}(\bar{Y})\right)$ is hard for VP under projections.

We use rigid and mutually incomparable graphs in the construction of $G_{m}$. Let $I:=$ $\left\{I_{0}, I_{1}, I_{2}\right\}$ be a fixed set of three connected, rigid and mutually incomparable graphs. Note that they are necessarily non-bipartite. Let $c_{I_{i}}=\left|V\left(I_{i}\right)\right|$. Choose an integer $c_{\max }>\max \left\{c_{I_{0}}, c_{I_{1}}, c_{I_{2}}\right\}$. Identify two distinct vertices $\left\{v_{\ell}^{0}, v_{r}^{0}\right\}$ in $I_{0}$, three distinct vertices $\left\{v_{\ell}^{1}, v_{r}^{1}, v_{p}^{1}\right\}$ in $I_{1}$, and three distinct vertices $\left\{v_{\ell}^{2}, v_{r}^{2}, v_{p}^{2}\right\}$ in $I_{2}$.

For every $m$ a power of 2 , we denote a complete (perfect) binary tree with $m$ leaves by $\mathrm{T}_{m}$. We construct a sequence of graphs $G_{m}$ (Fig. 11) from $\mathrm{T}_{m}$ as follows: first replace the root by the graph $I_{0}$, then all the nodes on a particular level are replaced by either $I_{1}$ or $I_{2}$ alternately (cf. Fig. 11). Now we add edges; suppose we are at a 'node' which is labeled $I_{i}$ and the left child and right child are labeled $I_{j}$, we add an edge between $v_{\ell}^{i}$ and $v_{p}^{j}$ in the left child, and an edge between $v_{r}^{i}$ and $v_{p}^{j}$ in the right child. Finally, to obtain $G_{m}$ we expand each added edge into a simple path with $c_{\max }$ vertices on it (cf. Fig. 1). That is, a left-edge connection between two incomparable graphs in the tree looks like, $I_{i}\left(v_{\ell}^{i}\right)-$ (path with $c_{\max }$ vertices) $-\left(v_{p}^{j}\right) I_{j}$.

Theorem 6. Over any field, the family of homomorphism polynomials $\left(f_{m}\right)$, with $f_{m}(\bar{Y})=$ $f_{G_{m}, H_{m}}(\bar{Y})$, where

- $G_{m}$ is defined as above (see Fig. 1), and

- $H_{m}$ is an undirected complete graph on poly $(m)$, say $m^{6}$, vertices, 


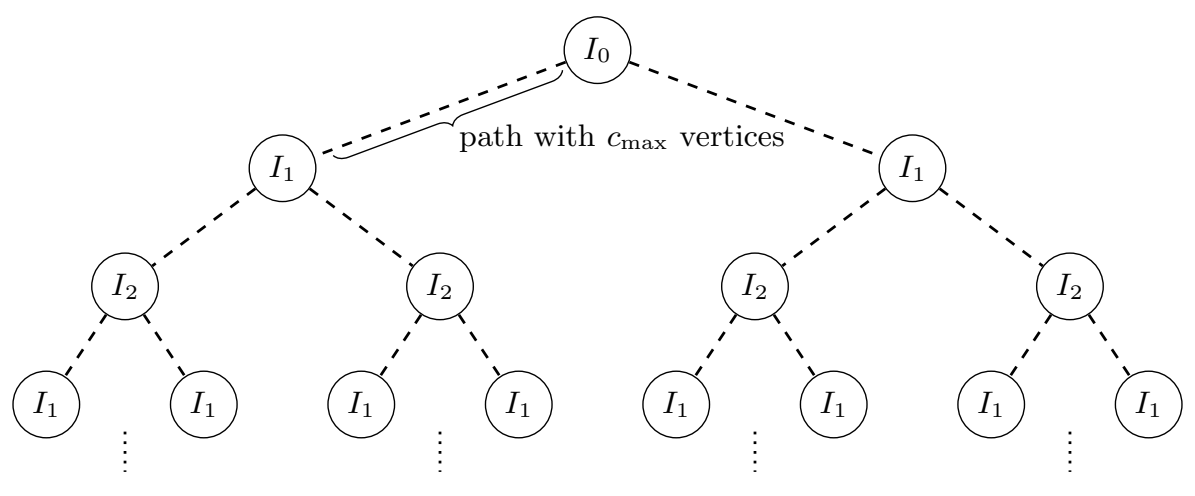

Fig. 1. The graph $G_{m}$.

is complete for VP under p-projections.

Proof. Membership in VP follows from Theorem [5,

We proceed with the hardness proof. The idea is to obtain the VP-complete universal polynomial from [23] as a projection of $f_{m}$. This universal polynomial is computed by a normal-form homogeneous circuit with alternating unbounded fanin-in + and bounded fanin $\times$ gates. We would like to put its parse trees in bijection with homomorphisms from $G$ to $H$. This becomes easier if we use an equivalent universal circuit in a nice normal form as described in [11]. The normal form circuit is multiplicatively disjoint; sub-circuits of $\times$ gates are disjoint (see [21]). This ensures that even though $C_{n}$ itself is not a formula, all its parse trees are already subgraphs of $C_{n}$ even without unwinding it into a formula.

Our starting point is the related graph $J_{n}^{\prime}$ in [11. The parse trees in $C_{n}$ are complete alternating unary-binary trees. The graph $J_{n}^{\prime}$ is constructed in such a way that the parse trees are now in bijection with complete binary trees. To achieve this, we "shortcut" the + gates, while preserving information about whether a subtree came in from the left or the right. For completeness sake we describe the construction of $J_{n}^{\prime}$ from [11].

We obtain a sequence of graphs $\left(J_{n}^{\prime}\right)$ from the undirected graphs underlying $\left(C_{n}\right)$ as follows. Retain the multiplication and input gates of $C_{n}$. Let us make two copies of each. For each retained gate, $g$, in $C_{n}$; let $g_{L}$ and $g_{R}$ be the two copies of $g$ in $J_{n}^{\prime}$. We now define the edge connections in $J_{n}^{\prime}$. Assume $g$ is a $\times$ gate retained in $J_{n}^{\prime}$. Let $\alpha$ and $\beta$ be two + gates feeding into $g$ in $C_{n}$. Let $\left\{\alpha_{1}, \ldots, \alpha_{i}\right\}$ and $\left\{\beta_{1}, \ldots, \beta_{j}\right\}$ be the gates feeding into $\alpha$ and $\beta$, respectively. Assume without loss of generality that $\alpha$ and $\beta$ feed into $g$ from left and right, respectively. We add the following set of edges to $J_{n}^{\prime}:\left\{\left(\alpha_{1 L}, g_{L}\right), \ldots,\left(\alpha_{i L}, g_{L}\right)\right\},\left\{\left(\beta_{1 R}, g_{L}\right), \ldots,\left(\beta_{j R}, g_{L}\right)\right\}$, $\left\{\left(\alpha_{1 L}, g_{R}\right), \ldots,\left(\alpha_{i L}, g_{R}\right)\right\}$ and $\left\{\left(\beta_{1 R}, g_{R}\right), \ldots,\left(\beta_{j R}, g_{R}\right)\right\}$. We now would like to keep a single copy of $C_{n}$ in these set of edges. So we remove the vertex root $_{R}$ and we remove the remaining spurious edges in following way. If we assume that all edges are directed from root towards leaves, then we keep only edges induced by the vertices reachable from root $t_{L}$ in this directed graph. In [11], it was observed that there is a one-to-one correspondence between parse trees of $C_{n}$ and subgraphs of $J_{n}^{\prime}$ that are rooted at root $_{L}$ and isomorphic to $\mathrm{T}_{2^{k(n)}}$.

We now transform $J_{n}^{\prime}$ using the set $I=\left\{I_{0}, I_{1}, I_{2}\right\}$. This is similar to the transformation we did to the balanced binary tree $\mathrm{T}_{m}$. We replace each vertex by a graph in $I ; \operatorname{root}_{L}$ gets $I_{0}$ 
and the rest of the layers get $I_{1}$ or $I_{2}$ alternately (as in Fig. 11). Edge connections are made so that a left/right child is connected to its parent via the edge $\left(v_{p}^{j}, v_{\ell}^{i}\right) /\left(v_{p}^{j}, v_{r}^{i}\right)$. Finally we replace each edge connection by a path with $c_{\max }$ vertices on it (as in Fig. 11), to obtain the graph $J_{n}$. All edges of $J_{n}$ are labeled 1, with the following exceptions: Every input node contains the same rigid graph $I_{i}$. It has a vertex $v_{p}^{i}$. Each path connection to other nodes has this vertex as its end point. Label such path edges that are incident on $v_{p}^{i}$ by the label of the input gate.

Let $m:=2^{k(n)}$. The choice of poly $(m)$ is such that $4 s_{n} \leqslant$ poly $(m)$, where $s_{n}$ is the size of $J_{n}$. The $\bar{Y}$ variables are set to $\{0,1, \bar{x}\}$ such that the non-zero variables pick out the graph $J_{n}$. From the observations of [11] it follows that for each parse tree $p$ - $\mathrm{\top}$ of $C_{n}$, there exists a homomorphism $\phi: G_{2^{k(n)}} \rightarrow J_{n}$ such that $\operatorname{mon}(\phi)$ is exactly equal to $\operatorname{mon}(p-\mathrm{T})$. By $\operatorname{mon}(\cdot)$ we mean the monomial associated with an object. We claim that these are the only valid homomorphisms from $G_{2^{k(n)}} \rightarrow J_{n}$. We observe the following properties of homomorphisms from $G_{2^{k(n)}} \rightarrow J_{n}$, from which the claim follows. In the following by a rigid-node-subgraph we mean a graph in $\left\{I_{0}, I_{1}, I_{2}\right\}$ that replaces a vertex.

( $i$ ) Any homomorphic image of a rigid-node-subgraph of $G_{2^{k(n)}}$ in $J_{n}$, cannot split across two mutually incomparable rigid-node-subgraphs in $J_{n}$. That is, there cannot be two vertices in a rigid subgraph of $G_{2^{k(n)}}$ such that one of them is mapped into a rigid subgraph say $n_{1}$, and the other one is mapped into another rigid subgraph say $n_{2}$. This follows because homomorphisms do not increase distance.

(ii) Because of $(i)$, with each homomorphic image of a rigid node $g_{i} \in G_{2^{k(n)}}$, we can associate at most one rigid node of $J_{n}$, say $n_{i}$, such that the homomorphic image of $g_{i}$ is a subgraph of $n_{i}$ and the paths (corresponding to incident edges) emanating from it. But such a subgraph has a homomorphism to $n_{i}$ itself: fold each hanging path into an edge and then map this edge into an edge within $n_{i}$. (For instance, let $\rho$ be a path hanging off $n_{i}$ and attached to $n_{i}$ at $u$, and let $v$ be any neighbour of $u$ within $n_{i}$. Mapping vertices of $\rho$ to $u$ and $v$ alternately preserves all edges and hence is a homomorphism.) Therefore, we note that in such a case we have a homomorphism from $g_{i} \rightarrow n_{i}$. By rigidity and mutual incomparability, $g_{i}$ must be the same as $n_{i}$, and this folded-path homomorphism must be the identity map. The other scenario, where we cannot associate any $n_{i}$ because $g_{i}$ is mapped entirely within connecting paths, is not possible since it contradicts nonbipartiteness of mutually-incomparable graphs.

Root must be mapped to the root: The rigidity of $I_{0}$ and Property $(i i)$ implies that $I_{0} \in G_{2^{k(n)}}$ is mapped identically to $I_{0}$ in $J_{n}$.

Every level must be mapped within the same level: The children of $I_{0}$ in $G_{2^{k(n)}}$ are mapped to the children of the root while respecting left-right behaviour. Firstly, the left child cannot be mapped to the root because of incomparability of the graphs $I_{1}$ and $I_{0}$. Secondly, the left child cannot be mapped to the right child (or vice versa) even though they are the same graphs, because the minimum distance between the vertex in $I_{0}$ where the left path emanates and the right child is $c_{\max }+1$ whereas the distance between the vertex in $I_{0}$ where the left path emanates and the left child is $c_{\max }$. So some vertex from the left child must be mapped into the path leading to the right child and hence the rest of the left child must be 
mapped into a proper subgraph of right child. But this contradicts rigidity of $I_{1}$. Continuing like this, we can show that every level must map within the same level and that the mapping within a level is correct.

\subsection{VBP-completeness}

Finally, we show that homomorphism polynomials are also rich enough to characterize computation by algebraic branching programs. Here we establish that there exists a $p$-family $\left(G_{k}\right)$ of undirected bounded path-width graphs such that the family $\left(f_{G_{k}, H_{k}}(\bar{Y})\right)$ is VBP-complete with respect to $p$-projections.

We note that for VBP-completeness under projections, the construction in [11] required directed graphs. In the undirected setting they could establish hardness only under linear p-projection, that too using 0-1 valued weights.

As before, we use rigid and mutually incomparable graphs in the construction of $G_{k}$. Let $I:=\left\{I_{1}, I_{2}\right\}$ be two connected, non-bipartite, rigid and mutually incomparable graphs. Arbitrarily pick vertices $u \in V\left(I_{1}\right)$ and $v \in V\left(I_{2}\right)$. Let $c_{I_{i}}=\left|V\left(I_{i}\right)\right|$, and $c_{\max }=\max \left\{c_{I_{1}}, c_{I_{2}}\right\}$. Consider the sequence of graphs $\mathrm{G}_{k}$ (Fig. 2); for every $k$, there is a simple path with $(k-$ $1)+2 c_{\max }$ edges between a copy of $I_{1}$ and $I_{2}$. The path is between the vertices $u \in V\left(I_{1}\right)$ and $v \in V\left(I_{2}\right)$. The path between vertices $a$ and $b$ in $\mathrm{G}_{k}$ contains $(k-1)$ edges.

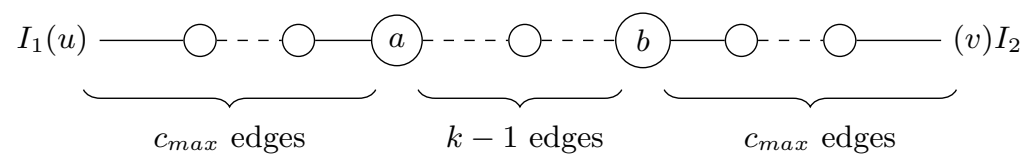

Fig. 2. The graph $\mathrm{G}_{k}$.

In other words, connect $I_{1}$ and $I_{2}$ by stringing together a path with $c_{\text {max }}$ edges between $u$ and $a$, a path with $k-1$ edges between $a$ and $b$, and a path with $c_{\text {max }}$ edges between $b$ and $v$.

Theorem 7. Over any field, the family of homomorphism polynomials $\left(f_{k}\right)$, where

- $G_{k}$ is defined as above (see Fig. Q),

- $H_{k}$ is the undirected complete graph on $O\left(k^{2}\right)$ vertices,

- $f_{k}(\bar{Y})=f_{G_{k}, H_{k}}(\bar{Y})$,

is complete for VBP with respect to p-projections.

Proof. Membership: It follows from Theorem 5 ,

Hardness: Let $\left(g_{n}\right) \in$ VBP. Without loss of generality, we can assume that $g_{n}$ is computable by a layered branching program of polynomial size such that the number of layers, $\ell$, is more than the width of the algebraic branching program.

Let $B_{n}^{\prime}$ be the undirected graph underlying the layered branching program $A_{n}$ for $g_{n}$. Let $B_{n}$ be the following graph: $I_{1}(u)-(s) B_{n}^{\prime}(t)-(v) I_{2}$, that is, $u \in I_{1}$ is connected to $s \in B_{n}^{\prime}$ 
via a path with $c_{\max }$ edges and $t \in B_{n}^{\prime}$ is connected to $v \in I_{2}$ via a path with $c_{\max }$ edges (cf. Fig. 2). The edges in $B_{n}^{\prime}$ inherits the weight from $A_{n}$, and the rest of the edges in $B_{n}$ have weight 1 .

Let us now consider $f_{\ell}$ when the variables on the edges of $H_{\ell}$ are instantiated to values in $\{0,1\}$ or variables of $g_{n}$ so that we obtain $B_{\ell}$ as a subgraph of $H_{\ell}$. We claim that a valid homomorphism from $\mathrm{G}_{\ell} \rightarrow B_{\ell}$ must satisfy the following properties:

(P1) $I_{1}$ in $\mathrm{G}_{\ell}$ must be mapped to $I_{1}$ in $B_{\ell}$ using the identity homomorphism, (P2) $I_{2}$ in $\mathrm{G}_{\ell}$ must be mapped to $I_{2}$ in $B_{\ell}$ using the identity homomorphism.

Assuming the claim, it follows that homomorphisms from $\mathrm{G}_{\ell} \rightarrow B_{\ell}$ are in one-to-one correspondence with $s$ - $t$ paths in $A_{n}$. In particular, the vertex $a \in \mathrm{G}_{\ell}$ is mapped to the vertex $s$ in $B_{\ell}$, and the vertex $b \in \mathrm{G}_{\ell}$ is mapped to the vertex $t$ in $B_{\ell}$. Also, the monomial associated with a homomorphism and its corresponding path are the same. Therefore, we have,

$$
f_{\mathrm{G}_{\ell}, B_{\ell}}=g_{n} .
$$

Since $\ell$ is polynomially bounded, we obtain VBP-completeness of $\left(f_{k}\right)$ over any field.

Let us now prove the claim. We first prove that a valid homomorphism from $\mathrm{G}_{\ell} \rightarrow B_{\ell}$ must satisfy the property (P1). There are three cases to consider.

- Case 1: Some vertex of $V\left(I_{1}\right) \subseteq V\left(G_{\ell}\right)$ is mapped to $u$ in $B_{\ell}$. Since homomorphisms cannot increase distances between two vertices, we conclude that $V\left(I_{1}\right)$ must be mapped within the subgraph $I_{1}(u)-(a)$. Suppose further that some vertex on the $(u)-(a)$ path other than $u$ is also in the homomorphic image of $V\left(I_{1}\right)$. Some neighbour of $u$ in $V\left(I_{1}\right) \subseteq V\left(B_{\ell}\right)$, say $u^{\prime}$, must also be in the homomorphic image, since otherwise we have a homomorphism from the non-bipartite $I_{1}$ to a path, a contradiction. But note that $I_{1}(u)-(a)$ has a homomorphism to $I_{1}$ : fold the $(u)-(a)$ path onto the edge $u-u^{\prime}$ in $I_{1}$. Hence, composing the two homomorphisms we obtain a homomorphism from $I_{1}$ to $I_{1}$ which is not surjective. This contradicts the rigidity of $I_{1}$. So in fact the homomorphism must map $V\left(I_{1}\right)$ from $G_{\ell}$ entirely within $I_{1}$ from $B_{\ell}$, and by rigidity of $I_{1}$, this must be the identity map.

- Case 2: Some vertex of $V\left(I_{1}\right) \subseteq V\left(G_{\ell}\right)$ is mapped to $v$ in $B_{\ell}$. Since homomorphisms cannot increase distances between two vertices, we conclude that $V\left(I_{1}\right)$ must be mapped within the subgraph $(b)-(v) I_{2}$. But note that $(b)-(v) I_{2}$ has a homomorphism to $I_{2}$ (fold the $(b)-(v)$ path onto any edge incident on $v$ within $I_{2}$ ). Hence, composing the two homomorphisms, we obtain a homomorphism from $I_{1}$ to $I_{2}$. This is a contradiction, since $I_{1}$ and $I_{2}$ were incomparable graphs to start with.

- Case 3: No vertex of $V\left(I_{1}\right) \subseteq V\left(G_{\ell}\right)$ is mapped to $u$ or $v$ in $B_{\ell}$. Then $V\left(I_{1}\right) \subseteq V\left(\mathrm{G}_{\ell}\right)$ must be mapped entirely within one of the following disjoint regions of $B_{\ell}$ : (a) $I_{1} \backslash\{u\}$, (b) bipartite graph between vertices $u$ and $v$, and (c) $I_{2} \backslash\{v\}$. But then we contradict rigidity of $I_{1}$ in the first case, non-bipartiteness of $I_{1}$ in the second case, and incomparability of $I_{1}$ and $I_{2}$ in the last.

In a similar way, we could also prove that a valid homomorphism from $\mathrm{G}_{\ell} \rightarrow B_{\ell}$ must satisfy the property (P2). 
In the above proof, we crucially used incomparability of $I_{1}$ and $I_{2}$ to rule out flipping an undirected path. It turns out that over fields of characteristic not equal to 2 , this is not crucial, since we can divide by 2 . We show that if the characteristic of the underlying field is not equal to 2 , then the sequence $\left(G_{k}\right)$ in the preceding theorem can be replaced by a sequence of simple undirected cycles of appropriate length. In particular, we establish the following result.

Theorem 8. Over fields of char $\neq 2$, the family of homomorphism polynomials $\left(f_{k}\right), f_{k}=$ $f_{G_{k}, H_{k}}$, where

- $G_{k}$ is a simple undirected cycle of length $2 k+1$ and,

- $H_{k}$ is an undirected complete graph on $(2 k+1)^{2}$ vertices,

is complete for VBP under p-projections.

Proof. Membership: As before, it follows from Theorem 5 ,

Hardness: Let $\left(g_{n}\right) \in$ VBP. Without loss of generality, we can assume that $g_{n}$ is computable by a layered branching program of polynomial size satisfying the following properties:

- The number of layers, $\ell \geqslant 3$, is odd; say $\ell=2 m+1$. So every path from $s$ to $t$ in the branching program has exactly $2 m$ edges.

- The number of layers, is more than the width of the algebraic branching program,

Let us consider $f_{m}$ when the variables on the edges of $H_{m}$ have been set to 0 , 1 , or variables of $g_{n}$ so that we obtain the undirected graph underlying the layered branching program $A_{n}$ for $g_{n}$ as a subgraph of $H_{m}$. Now change the weight of the $(s, t)$ edge from 0 to weight $y$, where $y$ is a new variable distinct from all the other variables of $g_{n}$. Call this modified graph $B_{m}$. Note that without the new edge, $B_{m}$ would be bipartite.

Let us understand the homomorphisms from $G_{m}$ to $B_{m}$. Homomorphisms from a simple cycle $C$ to a graph $\mathcal{G}$ are in one-to-one correspondence with closed walks of the same length in $\mathcal{G}$. Moreover, if the cycle $C$ is of odd length, the closed walk must contain a simple odd cycle of at most the same length. Therefore, the only valid homomorphism from $G_{m}$ to $B_{m}$ are walks of length $\ell=2 m+1$, and they all contain the edge $(s, t)$ with weight $y$. But the cycles of length $\ell$ in $B_{m}$ are in one-to-one correspondence with $s$ - $t$ paths in $A_{n}$. Each cycle contributes $2 \ell$ walks: we can start the walk at any of the $\ell$ vertices, and we can follow the directions from $A_{n}$ or go against those directions. Thus we have,

$$
f_{G_{m}, B_{m}}=(2(2 m+1)) \cdot y \cdot g_{n}=(2 \ell) \cdot y \cdot g_{n} .
$$

Let $p$ be the characteristic of the underlying field. If $p=0$, we substitute $y=(2 \ell)^{-1}$ to obtain $g_{n}$. If $p>2$, then $2 \ell$ has an inverse if and only if $\ell$ has an inverse. Since $\ell \geqslant 3$ is an odd number, either $p$ does not divide $\ell$ or it does not divide $\ell+2$. Hence, at least one of $\ell$, $\ell+2$ has an inverse. Thus $g_{n}$ is a projection of $f_{m}$ or $f_{m+1}$ depending on whether $\ell$ or $\ell+2$ has an inverse in characteristic $p$.

Since $\ell=2 m+1$ is polynomially bounded in $n$, we therefore show $\left(f_{k}\right)$ is VBP-complete with respect to $p$-projections over any field of characteristic not equal to 2 . 


\section{Conclusion}

In this paper, we have shown that over finite fields, five families of polynomials are intermediate in complexity between VP and VNP, assuming the PH does not collapse. Over rationals and reals, we have established that two of these families are provably not monotone $p$-projections of the permanent polynomials. Finally, we have obtained a natural family of polynomials, defined via graph homomorphisms, that is complete for VP with respect to projections; this is the first family defined independent of circuits and with such hardness. An analogous family is also shown to be complete for VBP.

Several interesting questions remain.

The definitions of our intermediate polynomials use the size $q$ of the field $\mathbb{F}_{q}$, not just the characteristic $p$. Can we find families of polynomials with integer coefficients, that are VNP-intermediate (under some natural complexity assumption of course) over all fields of characteristic $p$ ? Even more ambitiously, can we find families of polynomials with integer coefficients, that are VNP-intermediate over all fields with non-zero characteristic? at least over all finite fields? over fields $\mathbb{F}_{p}$ for all (or even for infinitely many) primes $p$ ?

Equally interestingly, can we find an explicit family of polynomials that is VNP-intermediate in characteristic zero?

A related question is whether there are any polynomials defined over the integers, that are VNP-intermediate over $\mathbb{F}_{q}$ (for some fixed $q$ ) but that are monotone $p$-projections of the permanent.

Can we show that the remaining intermediate polynomials are also not polynomial-sized monotone projections of the permanent? Do such results have any interesting consequences, say, improved circuit lower bounds?

\section{References}

[1] Noga Alon and Ravi B. Boppana. The monotone circuit complexity of Boolean functions. Combinatorica, $7(1): 1-22,1987$.

[2] David Avis and Hans Raj Tiwary. On the extension complexity of combinatorial polytopes. In Automata, Languages, and Programming - 40th International Colloquium, ICALP Part I, pages 57-68, 2013.

[3] Walter Baur and Volker Strassen. The complexity of partial derivatives. Theoretical Computer Science, 22(3):317 - 330, 1983.

[4] P. Bürgisser. Completeness and Reduction in Algebraic Complexity Theory, volume 7 of Algorithms and Computation in Mathematics. Springer, 2000.

[5] Peter Bürgisser. On the structure of Valiant's complexity classes. Discrete Mathematics $\&$ Theoretical Computer Science, 3(3):73-94, 1999.

[6] Peter Bürgisser. Cook's versus Valiant's hypothesis. Theoretical Computer Science, 235(1):71-88, 2000.

[7] Florent Capelli, Arnaud Durand, and Stefan Mengel. The arithmetic complexity of tensor contractions. In Symposium on Theoretical Aspects of Computer Science STACS, volume 20 of LIPIcs, pages 365-376, 2013.

[8] Marek Cygan, Fedor V. Fomin, Lukasz Kowalik, Daniel Lokshtanov, Dániel Marx, Marcin Pilipczuk, Michal Pilipczuk, and Saket Saurabh. Parameterized Algorithms. Springer, 2015.

[9] Nicolas de Rugy-Altherre. A dichotomy theorem for homomorphism polynomials. In Mathematical Foundations of Computer Science 2012, volume 7464 of LNCS, pages 308-322. Springer Berlin Heidelberg, 2012.

[10] Josep Díaz, Maria J. Serna, and Dimitrios M. Thilikos. Counting h-colorings of partial k-trees. Theoretical Computer Science, 281(1-2):291-309, 2002.

[11] Arnaud Durand, Meena Mahajan, Guillaume Malod, Nicolas de Rugy-Altherre, and Nitin Saurabh. Homomorphism polynomials complete for VP. In 34th Foundation of Software Technology and Theoretical Computer Science Conference, FSTTCS, pages 493-504, 2014. 
[12] Jack Edmonds. Paths, trees, and flowers. Canadian Journal of Mathematics, 17:449-467, 1965.

[13] Samuel Fiorini, Serge Massar, Sebastian Pokutta, Hans Raj Tiwary, and Ronald de Wolf. Exponential lower bounds for polytopes in combinatorial optimization. J. ACM, 62(2):17, 2015.

[14] M. R. Garey and David S. Johnson. Computers and Intractability: A Guide to the Theory of NP-Completeness. W. H. Freeman, 1979.

[15] Joshua A. Grochow. Monotone projection lower bounds from extended formulation lower bounds. arXiv:1510.08417 [cs.CC], 2015.

[16] Pavol Hell and Jaroslav Nešetřil. Graphs and homomorphisms. Oxford lecture series in mathematics and its applications. Oxford University Press, 2004.

[17] Mark Jerrum and Marc Snir. Some exact complexity results for straight-line computations over semirings. J. ACM, 29(3):874-897, 1982.

[18] Stasys Jukna. Why is Hamilton Cycle so different from Permanent? http://cstheory.stackexchange.com/questions/27496/why-is-hamiltonian-cycle-so-different-from-permanent, 2014.

[19] Richard M Karp and Richard Lipton. Turing machines that take advice. L'enseignement mathématique, 28(2):191-209, 1982.

[20] Richard E. Ladner. On the structure of polynomial time reducibility. J. ACM, 22(1):155-171, 1975.

[21] Guillaume Malod and Natacha Portier. Characterizing Valiant's algebraic complexity classes. Journal of Complexity, 24(1):16-38, 2008.

[22] Stefan Mengel. Characterizing arithmetic circuit classes by constraint satisfaction problems. In Automata, Languages and Programming, volume 6755 of LNCS, pages 700-711. Springer Berlin Heidelberg, 2011.

[23] Ran Raz. Elusive functions and lower bounds for arithmetic circuits. Theory of Computing, 6:135-177, 2010.

[24] A. A. Razborov. Lower bounds on the monotone complexity of some Boolean functions. Dokl. Akad. Nauk SSSR, 281(4):798-801, 1985.

[25] A.A. Razborov. Lower bounds on monotone complexity of the logical permanent. Mathematical notes of the Academy of Sciences of the USSR, 37(6):485-493, 1985.

[26] Thomas Rothvoß. The matching polytope has exponential extension complexity. In Symposium on Theory of Computing, STOC 2014, New York, NY, USA, May 31 - June 03, 2014, pages 263-272, 2014.

[27] Janos Simon. On the difference between one and many (preliminary version). In Automata, Languages and Programming, Fourth Colloquium, University of Turku, Finland, July 18-22, 1977, Proceedings, pages 480-491, 1977.

[28] Leslie G. Valiant. Completeness classes in algebra. In Symposium on Theory of Computing STOC, pages 249-261, 1979.

[29] Leslie G. Valiant, Sven Skyum, S. Berkowitz, and Charles Rackoff. Fast parallel computation of polynomials using few processors. SIAM Journal on Computing, 12(4):641-644, 1983. 